\title{
Machine learning derived risk prediction of anorexia nervosa
}

\author{
Yiran Guo ${ }^{1 *}$, Zhi Wei ${ }^{2}$, Brendan J. Keating ${ }^{1,3}$, The Genetic Consortium for Anorexia Nervosa, The Wellcome Trust \\ Case Control Consortium 3, Price Foundation Collaborative Group and Hakon Hakonarson 1,3*
}

\begin{abstract}
Background: Anorexia nervosa (AN) is a complex psychiatric disease with a moderate to strong genetic contribution. In addition to conventional genome wide association (GWA) studies, researchers have been using machine learning methods in conjunction with genomic data to predict risk of diseases in which genetics play an important role.

Methods: In this study, we collected whole genome genotyping data on 3940 AN cases and 9266 controls from the Genetic Consortium for Anorexia Nervosa (GCAN), the Wellcome Trust Case Control Consortium 3 (WTCCC3), Price Foundation Collaborative Group and the Children's Hospital of Philadelphia (CHOP), and applied machine learning methods for predicting AN disease risk. The prediction performance is measured by area under the receiver operating characteristic curve (AUC), indicating how well the model distinguishes cases from unaffected control subjects.

Results: Logistic regression model with the lasso penalty technique generated an AUC of 0.693, while Support Vector Machines and Gradient Boosted Trees reached AUC's of 0.691 and 0.623, respectively. Using different sample sizes, our results suggest that larger datasets are required to optimize the machine learning models and achieve higher AUC values.

Conclusions: To our knowledge, this is the first attempt to assess AN risk based on genome wide genotype level data. Future integration of genomic, environmental and family-based information is likely to improve the AN risk evaluation process, eventually benefitting AN patients and families in the clinical setting.
\end{abstract}

Keywords: Anorexia nervosa, Machine learning, Genome wide association, Risk prediction, Genotyping

\section{Background}

Anorexia nervosa (AN) is a complex eating disorder with psychiatric manifestations, including strong obsessive concern about gaining weight, twisted self-depiction towards body shape and eating, and extremely low food intake resulting in below-average body mass index [1-3]. The estimated prevalence of AN in the general population is $1 \%$ [4] and it is sex-biased, with an estimated female to male ratio of $10: 1[1,3]$ and many patients being young women. Common comorbid psychiatric disorders include major depression disorder and anxiety disorders [5-9]. Among all psychiatric disorders, AN has one of the highest mortality rates [10-16]. However, interventions for AN have shown limited success and the hospitalization

\footnotetext{
* Correspondence: guoy@email.chop.edu; hakonarson@email.chop.edu ${ }^{1}$ The Center for Applied Genomics, Abramson Research Center, The Children's Hospital of Philadelphia, Philadelphia, PA 19104, USA

Full list of author information is available at the end of the article
}

for weight regain is time consuming and expensive [17-19]. Altogether, AN incurs serious physical, psychological, familial and social toll to the modern world.

Recent efforts have shown that genetics plays an important role in AN susceptibility with heritability estimates from twin studies are as high as $84 \%$ [20-26]. The inheritance is complex and multiple genes/loci are potentially involved, especially those in the dopamine pathway [27-29], weight/BMI related genes [30-33] and cholesterol metabolism regulatory pathway genes [34]. Three Genome Wide Association (GWA) studies [35-37] have been published without identifying an AN associated marker at genomewide significance level of $P$ value $<5 \mathrm{E}-8$. Nevertheless, several genome wide marginal results have been reported, suggesting larger sample size and/or denser genotyping or high throughput parallel sequencing may be required to unveil the genetic underpinnings of AN. 
Recently, machine learning based risk prediction methods using genotyping data have gained momentum in relation with GWA studies in complex disease [38-46], making an important contribution towards the promise of personalized medicine [47-49]. Here, we have organized the largest AN cohort so far [37], constructed machine learning models using GWA microarray data [47, 50], and applied the model to testing data set to evaluate the model's performance by the area under the receiver operating characteristic curve (AUC) $[47,51,52]$. AUC is a value between 0.5 and 1 that assesses how well the model can distinguish cases from unaffected controls, with the higher number indicating better discriminative power. To our knowledge, this is the first attempt to assess AN disease risk using genetic information alone and the results, together with further investigation into AN genetics, would be anticipated to contribute to early diagnosis and allow for preventive interventions of $\mathrm{AN}$ in the future.

\section{Methods}

\section{Ethics and consent}

Local ethical approvals were granted for each participating study. For participants under the age of 16, written informed consent from their parents were obtained; for participants over the age of 16, written informed consent from themselves were obtained. Full names and locations of the ethic committees can be found in the end of the Additional file 1.

\section{Participating studies, genotyping and phenotyping}

We combined individual level genotypic and phenotypic data from a total of 16 datasets in the Genetic Consortium for Anorexia Nervosa (GCAN)/Wellcome Trust Case Control Consortium 3 (WTCCC3), The Price Foundation Collaborative Group [53-55] and Children's Hospital of Philadelphia (CHOP; Table 1). The final dataset encompassed 3940 cases and 9266 controls. Phenotyping details, genotyping approaches and quality control are described elsewhere previously [37]. In brief, all cases were female older than 9 years and met DSM-IV [1] (the Diagnostic and Statistical Manual of Mental Disorders, $4^{\text {th }}$ Edition) diagnostic criteria (amenorrhea criterion not required). Genotyping was done using Illumina microarrays, followed by quality control and imputation (as described previously in ref. [37]). We also collated 5087 disease free controls from Center for Applied Genomics at CHOP, which have been successfully used in previous neurodevelopmental/ psychiatric GWA studies [56-59].

\section{Logistic regression model}

The entire dataset was randomly partitioned into three equal parts using Fisher-Yates permutation [60], without specifying case/control ratio. We then took a three step
Table 1 Sample sizes of participating studies

\begin{tabular}{lll}
\hline Country & Cases & Controls \\
\hline Canada & 54 & - \\
Czech Republic & 72 & - \\
Finland & 131 & 404 \\
France & 293 & - \\
Germany & 475 & - \\
Greece & 70 & - \\
Italy-North & 203 & - \\
Italy-South & 75 & - \\
Netherlands & 348 & - \\
Norway & 82 & - \\
Poland & 175 & - \\
Spain & 186 & - \\
Sweden & 39 & - \\
UK & 213 & - \\
USA & 491 & - \\
USA-CHOP & 1033 & 8862 \\
Total & 3940 & 9266 \\
\hline & & \\
\hline & &
\end{tabular}

procedure to conduct the logistic regression (LR) prediction, including a) predictor pre-selection in the first subset of data (i.e. fold1), b) model training with cross-validation in the second subset (fold2) and c) model testing and assessment in the third part (fold3). As information for model training and testing were randomly drawn from the same collection of data, any population stratification in fold3 is already accounted for in the model during the training process in fold2 [61].

In the pre-selection stage, a GWA study was conducted in fold1 using PLINK [62] with conventional settings of maximal per-SNP missingness of $1 \%$, maximal per-individual missingness of $5 \%$, minimally allowed minor allele frequency of $1 \%$, minimally allowed Hardy-Weinberg equilibrium test $P$ value of $1 \mathrm{E}-6$. Then we retained SNPs with genome-wide case-control association test $P$ value $<1 \mathrm{E}-3$ to the next stage. Next we employed lasso regularized LR model with ten-fold cross validation in R package 'glmnet' [63] (http://cran.r-project.org/web/ packages/glmnet/index.html) in fold2 data. A grid of lambda values (the regularization parameter in the model to reduce overfitting) are computed for the lasso penalty and AUC was measured to assess the performance. At the third stage, the model trained on fold2 data was then tested on fold3 data, and we calculated its AUC.

The procedure was repeated ten times using randomly shuffled datasets (Additional file 1: Table S1). Different sample sizes with randomized reruns were also examined to evaluate sample size effects to model fitting. 


\section{Support Vector Machine and Gradient Boosted Trees} In order to compare different machine learning techniques, Support Vector Machines (SVM) with RBF kernels and default parameters ( $\mathrm{R}$ package 'e1071'; http:// cran.r-project.org/web/packages/e1071/index.html) and Gradient Boosted Trees with default parameters ( $\mathrm{R}$ package 'gbm'; http://cran.r-project.org/web/packages/ $\mathrm{gbm} /$ index.html) approaches were also used thereafter to train models within fold2 and assess the performance within fold3.

\section{Results}

\section{Logistic regression model applied to the dataset}

After combining 16 datasets (Table 1), imputed genotyping data were collated for a total of 3940 cases and 9266 controls each at up to 317481 SNPs. We performed GWA scan in a subset of the cohort (the pre-selection dataset or fold1), which contains 1289 AN cases and 3113 healthy controls (Additional file 1: Table S1). Considering that currently no single marker has been found to be associated with AN at the level of $P$ value $<5 \mathrm{E}-8$ in any GWA study, we used SNPs with a less stringent threshold of $P$ value $<1 \mathrm{E}-3$, instead of the entire SNP list, for subsequent machine learning calculations. A total of 1486 SNPs were retained according to this criterion after quality control (Additional file 1: Table S1) and were utilized in LR model training and cross-validation. In the second subset of data with 1341 cases and 3061 controls (the model training dataset or fold2) where we did ten-fold cross-validation, the penalized LR with $L_{1}$ penalty [63] (the lasso) generated a model of 273 SNPs (Additional file 1: Table S1), with an AUC of 0.673 and regularization penalty parameter (lambda) of 0.00954 (Fig. 1). Subsequently we fitted this model to the third subset of 1310 cases and 3092 controls (the testing dataset or fold3), and the result indicated an AUC of 0.693 (Additional file 1: Table S1, Fig. 1 and

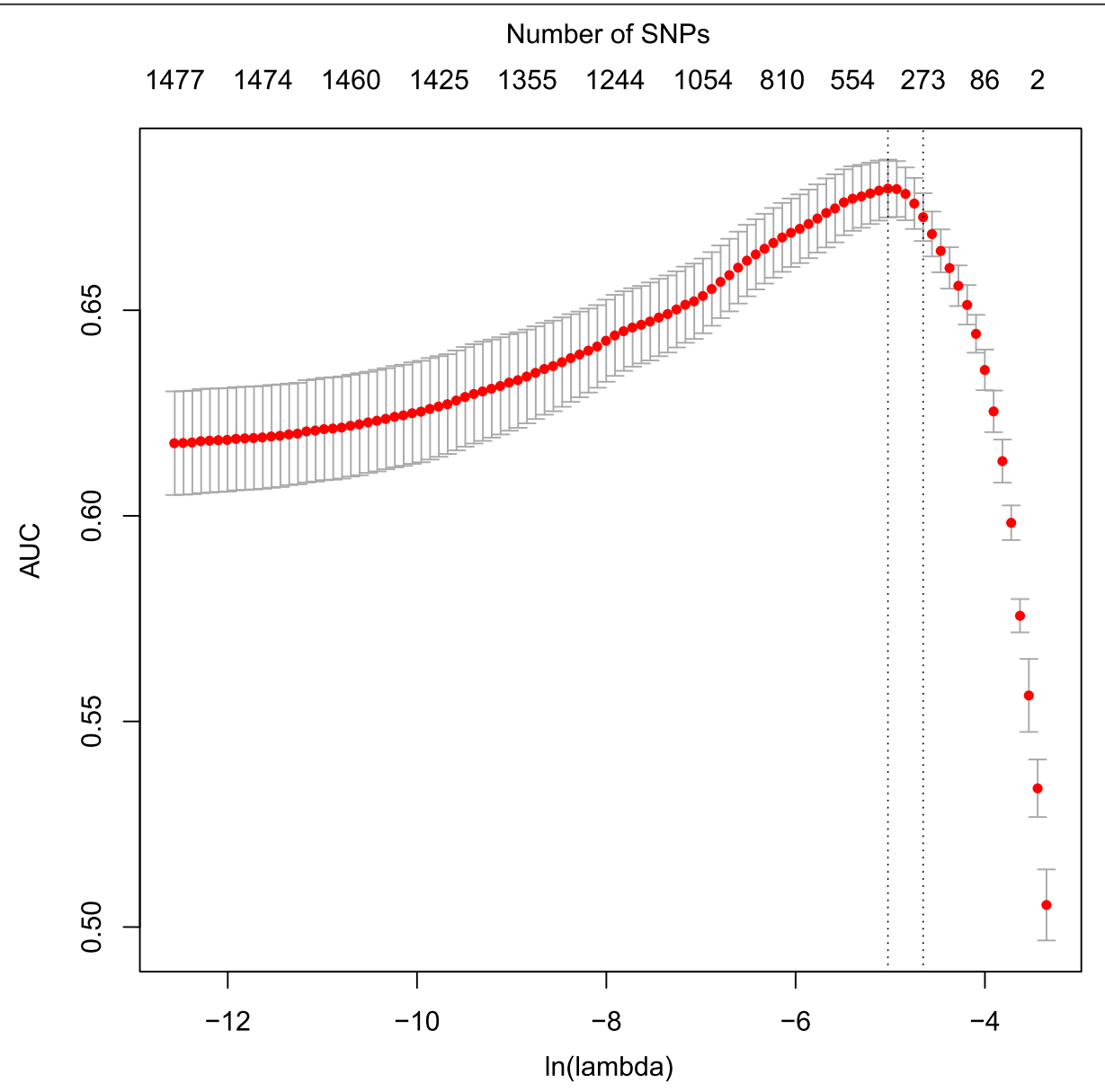

Fig. 1 Logistic regression model with ten-fold validation. By harnessing L1 penalty (the lasso), we further removed irrelevant SNPs in fold2 after the preselection step in fold1. Smaller lambda (the penalty parameter) values correspond to fewer SNPs removed, and numbers on the top of the plot indicate how many SNPS survived with respect to specific lambdas as X-axis (natural logarithm scale). We estimated the mean and standard error (SE) for AUCs across 100 different lambda values, and reported the largest lambda such that AUC is within 1 SE of the optimum (the left vertical dashed line shows the lambda with maximum of AUC, while the right vertical dashed line shows the lambda with AUC being within 1 SE of that maximum). The optimal 10-fold cross-validated AUCs on fold 2 data was 0.673 with regularization parameter lambda of 0.00954 
Additional file 2: Figure S1). We randomly shuffled the entire dataset nine more times and the results were similar for all runs (Additional file 1: Table S1). By using a 0.5 cutoff to the linearly calculated classifier output, we also calculated sensitivity and specificity in fold3, with values of $11 \%$ and $97 \%$, respectively.

\section{Sample size effects to the model}

We evaluated effects of sample sizes to the LR model performance. First, different percentages of fold2 samples were used for model training and the same fold3 dataset was fitted to measure the AUC values. We found a clear trend that AUC increases as the training population grows (Fig. 2 and Additional file 1: Table S2), which is consistent with the case of Inflammatory Bowel Disease (IBD) [44]. Results of ten randomized reruns showed significant differences between AUC's of smaller training sample sizes and that of the original $100 \%$ dataset $(P$ values $<4.2 \mathrm{E}-3$; Additional file 1: Table S2). We also experimented adding more samples from fold3 to fold2, in order to assess the behavior of the AUC trend in the situation of expanded training datasets. As shown in Fig. 3, AUC continues to increase along with the training dataset, especially when the training sample size is above 1.5 times of the original ( $P$ values $<=0.036$; Additional file 1: Table S3), despite larger variation from ten randomized reruns.

\section{Comparison with other machine learning methods}

We also explored two other widely used machine learning methods, Support Vector Machines (SVM) and Gradient Boosted Trees (GBT), and implemented them on our dataset following ten randomized repeats. While SVM provided similar performance in terms of AUC ( $P$ value $=0.099$; Additional file 1: Table S1), GBT was significantly inferior to the LR method we used $(P$ value $=$ 6.9E-10; Additional file 1: Table S1).

\section{Discussion}

We assessed AN disease risk using genome-wide SNP data by machine learning approaches on the largest AN cohort yet studied, representing one of the first applications of this kind in a complex psychiatric disorder [64,

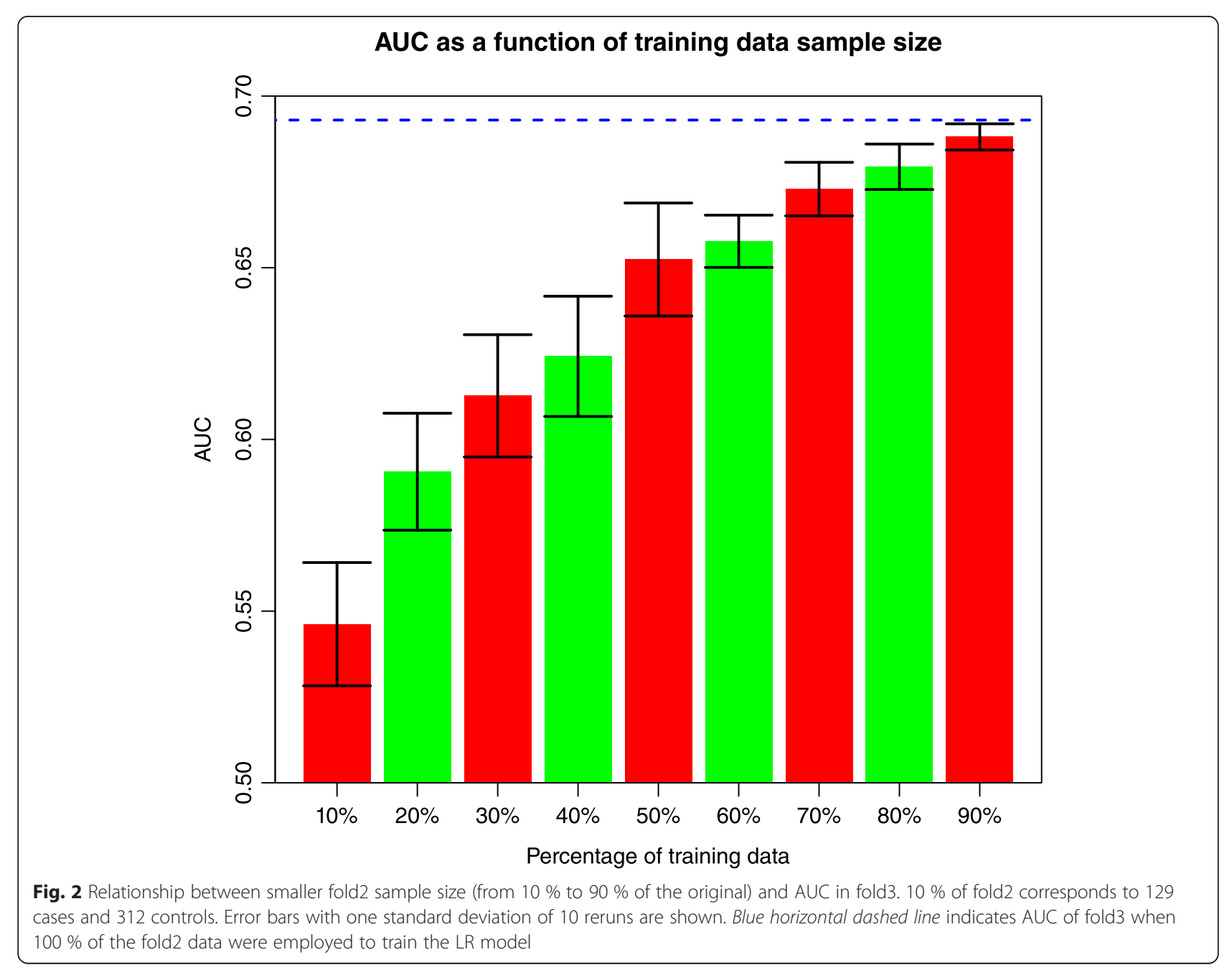




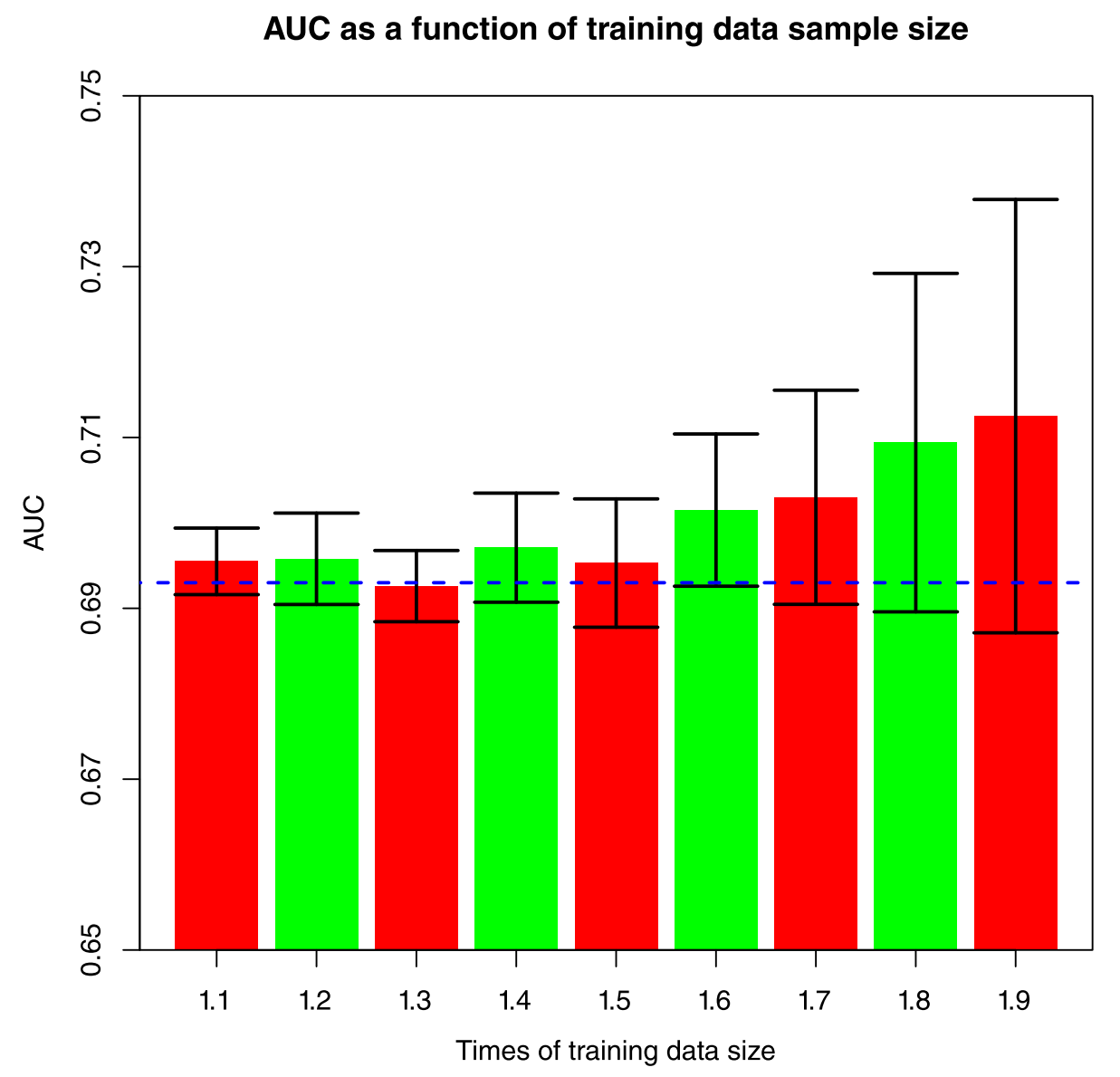

Fig. 3 Relationship between training dataset with more samples and AUC in the model testing dataset, when moving samples from fold 3 to fold2. $10 \%$ of fold2 corresponds to 129 cases and 312 controls. Error bars with one standard deviation of 10 reruns are shown. Blue horizontal dashed line indicates AUC of fold 3 when $100 \%$ of the fold 2 data were employed to train the LR model

65]. Our strategy follows a recent paper [66] with the basic idea that when data dimensionality is much larger than sample size, it is suggested to first do dimension reduction using a fast and simple method (e.g. univariate test), followed by some well-developed lower dimensional methods (e.g. lasso, Dantzig selector etc.). This can yield more accurate estimation as shown by our recent study [44]. After partitioning the cohort into three equal folds, we pre-selected SNPs in fold1 by filtering out those with genome-wide genetic association test $P$ value $>=1 \mathrm{E}-3$, trained the model in fold 2 using LR with $L_{1}$ penalty and cross-validation, and fit the model in fold3, achieving a discriminative measure AUC of 0.693 . We used AUC mainly because this is a case-control study which makes it hard, if possible, to measure calibration accuracy. More discussion can be found in ref. [67] regarding model accuracy. With more experiments on sample size adjustment, we discovered that the AUC for AN risk prediction was similarly sample size sensitive as other complex disorders like IBD [44], suggesting that larger sample sizes are needed to improve the machine learning models. We also assessed the posterior probability generated with the LR model in fold3, and the sensitivity and specificity were $11 \%$ and $97 \%$, respectively.

Our previous disease risk prediction efforts for type 1 diabetes (T1D) [39] and IBD [44] showed higher AUC as well as sensitivity/specificity values, which is consistent with, and can be explained by the fact that through successful GWA studies, genetic markers with significant association (e.g., $P$ value $<5 \mathrm{E}-8$ ) have been identified for both T1D and IBD, allowing for high performance machine learning models to discriminate cases from unaffected controls based on genotypic information. With regard to T1D [68], more than 40 genomic regions have been reported to be associated with the disease, and the human HLA genes are on the top of the list with $P$ values $<4 \mathrm{E}-136$. For IBD [69], more than 160 disease associated loci have been identified and markers in the IL23R region have $\mathrm{P}$ values $<1 \mathrm{E}-160$. For both $\mathrm{T} 1 \mathrm{D}$ and IBD, large sample sizes with approximately 10,000 patients in each case and significant contribution from the 
MHC region, are responsible for these successes and consequent superior disease risk prediction. Therefore with data from more AN samples in addition to the current 3940, it is highly likely that we will see better results for both the GWA effort and machine learning based risk prediction. Research into schizophrenia genetics provides a similar example in which large datasets led to the breakthrough of 128 independent genome-wide association signals [70] following identification of marginal hits with a few thousand cases in early stages [71].

In this report, we compared multiple machine learning methods LR, SVM and GBM. Results suggested that LR and SVM are similar in terms of AUC values, while GBM showed lower performance. From a practical perspective, the LR results are promising and LR models are easier to interpret and construct than SVM, although the current result requires improvement if genotype based AN risk prediction is to be used clinically. GBT is good at capturing interacting effects and may not be optimal when true models are linear. We have many SNPs typed thus it is likely interacting SNPs, if any, may already be interrogated well by a single SNP. In addition, here the number of SNPs is much larger than the sample size; a simple linear model may be more robust to over-fitting than assuming complex tree structures. We also tried to use random forests (RF) as well, but due to high time complexity and slow convergence this methods was excluded from the analysis. Further investigation is required to assess the performance of RF. Moreover, we can compare these methods with different parameter combinations and settings when a larger cohort of AN samples are available.

We shuffled LR model 10 times and got variable number of SNPs as predictors, with the observation that three SNPs are always in the shuffles (rs9982741, rs6092077 and rs2230513), and two SNPs (rs10250561 and rs16835204) are in 9 of the 10 shuffles. Those SNPs have the highest $\mathrm{p}$ values (from $4.39 \mathrm{E}-6$ to $3.05 \mathrm{E}-4$ ) in the fold1 GWA study. In light of AN's high heritability [20-26] and current lack of genome-wide significant markers [35-37], we anticipate that collating, phenotyping, genotyping and possibly sequencing more AN cases will reveal more strongly associated SNPs (thus serving as representative predictors), and greatly improve the performance of the machine learning models with higher specificity and sensitivity, which could be highly useful in a clinical setting. More complicated models including copy number variation, rare variants and even environmental factors will also lead to better performance. This and the discovery of significantly associated genomic loci or other biomarkers, will bring us closer to the goal of individualized medicine through early diagnosis and intervention for AN.

\section{Conclusion}

Using machine learning techniques, here we present the first AN risk prediction study based on genome wide genotype data. Our results indicated higher performances of LR and SVM as opposed to GBT, with the greatest discriminative value AUC being 0.693 for the linear model. In addition, we showed that larger sample sizes can improve the machine learning risk prediction outcome, urging expanded AN case collection through international collaboration. With more genomic and other data in a greater sample pool, our study and the motheds we used will serve as the first step toward genomic screening of AN risk in a clinical setting.

\section{Availability of supporting data}

Anorexia nervosa case summary statistics can be found at the PGC website (https://www.med.unc.edu/pgc/ downloads).

\section{Additional files}

Additional file 1 Table S1. Performance of logistic regression model in 10 random shuffles. Table S2. AUCs for fraction of the training dataset (from $10 \%$ to $90 \%$ of the original), after rerunning 10 times. Table S3. AUCs of different size for training dataset (from $10 \%$ more to $90 \%$ more than the original, randomly selected from fold3), after rerunning 10 times. Member lists of the Genetic Consortium for Anorexia Nervosa (GCAN)/the Wellcome Trust Case Control Consortium 3 (WTCCC 3)/the Price Foundation Collaborative Group. Supplementary acknowledgements. Ethic committee information. (DOCX 45 kb)

Additional file 2: Figure S1. ROC curve for linear regression model in shuffle 1. (PDF $17 \mathrm{~kb}$ )

\section{Abbreviations}

AN: Anorexia Nervosa; AUC: Area Under the receiver operating characteristic (ROC) Curve; GBT: Gradient Boosted Trees; GWA: genome wide association; IBD: Inflammatory Bowel Disease; LR: logistic regression; SNP: Single Nucleotide Polymorphism; SVM: Support Vector Machine; T1D: Type I Diabetes.

\section{Competing interests}

The authors declare that they have no competing interests.

\section{Authors' contributions}

YG, BJK and $\mathrm{HH}$ conceived of the study, and participated in its design and coordination. YG and ZW carried out the data analysis. YG drafted the manuscript. All authors read and approved the final manuscript.

\section{Acknowledgement}

Yiran Guo is funded by the 2011-2014 Davis Foundation Postdoctoral Fellowship Program in Eating Disorders Research Award. Genome-wide genotyping for CHOP samples was funded by an Institute Development Award to the Center for Applied Genomics from CHOP. Genotyping for the GCAN/WTCCC3 samples was supported by the Wellcome Trust, via The Wellcome Trust Case Control Consortium 3 project (WT090355/A/09/Z, WT090355/B/09/Z). We acknowledge the UK Medical Research Council and Wellcome Trust for funding the collection of DNA for The British 1958 Birth Cohort (MRC grant G0000934, Wellcome Trust grant 068545/Z/02). We acknowledge use of DNA from The UK Blood Services collection of Common Controls (UKBS collection), funded by Wellcome Trust grant 076113/C/04/Z, by Wellcome Trust/Juvenile Diabetes Research Foundation grant 061858 and by the National Institute of Health Research of England. The study was additionally funded through the Electronic Medical Records and Genomics (eMERGE) Network (U01 HG006830) by National Human Genome Research Institute 
of National Institutes of Health, and also funded by donation from the Kurbert Family. Other funding information can be found in the supplementary acknowledgements.

Full list of members of The Genetic Consortium for Anorexia Nervosa (GCAN), The Wellcome Trust Case Control Consortium 3 (WTCCC 3) and Price Foundation Collaborative Group can be found in the Additional file 1.

\section{Author details}

${ }^{1}$ The Center for Applied Genomics, Abramson Research Center, The Children's Hospital of Philadelphia, Philadelphia, PA 19104, USA. ²Department of Computer Science, New Jersey Institute of Technology, Newark, NJ 07102, USA. ${ }^{3}$ Department of Pediatrics, School of Medicine University of Pennsylvania, Philadelphia, PA 19104, USA.

\section{Received: 23 May 2015 Accepted: 15 January 2016}

\section{Published online: 20 January 2016}

\section{References}

1. American Psychiatric Association. Diagnostic and Statistical Manual of Mental Disorders. 4th ed. American Psychiatric Publishing, Incorporated; 1994.

2. Klump KL, Bulik CM, Kaye WH, Treasure J, Tyson E. Academy for eating disorders position paper: eating disorders are serious mental illnesses. Int J Eat Disord. 2009;42:97-103.

3. American Psychiatric Association. Diagnostic and Statistical Manual of Mental Disorders. 5th ed. Arlington, Virginia, United States: American Psychiatric Publishing, Incorporated; 2013.

4. Hudson Jl, Hiripi E, Pope Jr HG, Kessler RC. The prevalence and correlates of eating disorders in the National Comorbidity Survey Replication. Biol Psychiatry. 2007;61:348-58.

5. Sharp CW, Freeman CP. The medical complications of anorexia nervosa. Br J Psychiatry. 1993;162:452-62.

6. Godart NT, Flament MF, Perdereau F, Jeammet P. Comorbidity between eating disorders and anxiety disorders: a review. Int J Eat Disord. 2002;32: 253-70.

7. Kaye WH, Bulik CM, Thornton L, Barbarich N, Masters K. Comorbidity of anxiety disorders with anorexia and bulimia nervosa. Am J Psychiatry. 2004; 161:2215-21.

8. Katzman DK. Medical complications in adolescents with anorexia nervosa: a review of the literature. Int J Eat Disord. 2005;37 Suppl:S52-59. discussion S87-59.

9. Fernandez-Aranda F, Pinheiro AP, Tozzi F, Thornton LM, Fichter MM, Halmi $K A$, et al. Symptom profile of major depressive disorder in women with eating disorders. Aust N Z J Psychiatry. 2007;41:24-31.

10. Sullivan PF. Mortality in anorexia nervosa. Am J Psychiatry. 1995;152:1073-4.

11. Zipfel S, Lowe B, Reas DL, Deter HC, Herzog W. Long-term prognosis in anorexia nervosa: lessons from a 21-year follow-up study. Lancet. 2000; 355:721-2.

12. Birmingham $\mathrm{CL}$, Su J, Hlynsky JA, Goldner EM, Gao M. The mortality rate from anorexia nervosa. Int J Eat Disord. 2005;38:143-6.

13. Millar HR, Wardell F, Vyvyan JP, Naji SA, Prescott GJ, Eagles JM. Anorexia nervosa mortality in Northeast Scotland, 1965-1999. Am J Psychiatry. 2005; 162:753-7

14. Papadopoulos FC, Ekbom A, Brandt L, Ekselius L. Excess mortality, causes of death and prognostic factors in anorexia nervosa. Br J Psychiatry. 2009; 194:10-7.

15. Attia E. Anorexia nervosa: current status and future directions. Annu Rev Med. 2010;61:425-35.

16. Arcelus J, Mitchell AJ, Wales J, Nielsen S. Mortality rates in patients with anorexia nervosa and other eating disorders. A meta-analysis of 36 studies. Arch Gen Psychiatry. 2011;68:724-31.

17. Mckenzie JM, Joyce PR. Hospitalization for Anorexia-Nervosa. Int J Eat Disord. 1992;11:235-41.

18. Krauth $\mathrm{C}$, Buser $\mathrm{K}$, Vogel $\mathrm{H}$. How high are the costs of eating disorders - anorexia nervosa and bulimia nervosa - for German society? Eur J Health Econ. 2002;3: 244-50.

19. Bulik CM, Berkman ND, Brownley KA, Sedway JA, Lohr KN. Anorexia nervosa treatment: a systematic review of randomized controlled trials. Int J Eat Disord. 2007:40:310-20.

20. Lilenfeld LR, Kaye WH, Greeno CG, Merikangas KR, Plotnicov K, Pollice C, et al. A controlled family study of anorexia nervosa and bulimia nervosa: psychiatric disorders in first-degree relatives and effects of proband comorbidity. Arch Gen Psychiatry. 1998;55:603-10.

21. Strober M, Freeman R, Lampert C, Diamond J, Kaye W. Controlled family study of anorexia nervosa and bulimia nervosa: evidence of shared liability and transmission of partial syndromes. Am J Psychiatry. 2000;157: 393-401.

22. Wade TD, Bulik CM, Neale M, Kendler KS. Anorexia nervosa and major depression: shared genetic and environmental risk factors. Am J Psychiatry. 2000;157:469-71.

23. Klump KL, Miller KB, Keel PK, McGue M, lacono WG. Genetic and environmental influences on anorexia nervosa syndromes in a population-based twin sample. Psychol Med. 2001;31:737-40.

24. Kortegaard LS, Hoerder K, Joergensen J, Gillberg C, Kyvik KO. A preliminary population-based twin study of self-reported eating disorder. Psychol Med. 2001;31:361-5.

25. Bulik CM, Sullivan PF, Tozzi F, Furberg H, Lichtenstein P, Pedersen NL. Prevalence, heritability, and prospective risk factors for anorexia nervosa. Arch Gen Psychiatry. 2006;63:305-12.

26. Bulik CM, Thornton LM, Root TL, Pisetsky EM, Lichtenstein P, Pedersen NL. Understanding the relation between anorexia nervosa and bulimia nervosa in a Swedish national twin sample. Biol Psychiatry. 2010;67:71-7.

27. Bergen AW, van den Bree MB, Yeager M, Welch R, Ganjei JK, Haque K, et al. Candidate genes for anorexia nervosa in the 1p33-36 linkage region: serotonin 1D and delta opioid receptor loci exhibit significant association to anorexia nervosa. Mol Psychiatry. 2003;8:397-406.

28. Brown KM, Bujac SR, Mann ET, Campbell DA, Stubbins MJ, Blundell JE. Further evidence of association of OPRD1 \& HTR1D polymorphisms with susceptibility to anorexia nervosa. Biol Psychiatry. 2007;61:367-73.

29. Bergen AW, Yeager M, Welch RA, Haque K, Ganjei JK, van den Bree MB, et al. Association of multiple DRD2 polymorphisms with anorexia nervosa. Neuropsychopharmacology. 2005;30:1703-10.

30. Ribases M, Gratacos M, Fernandez-Aranda F, Bellodi L, Boni C, Anderluh $M$, et al. Association of BDNF with anorexia, bulimia and age of onset of weight loss in six European populations. Hum Mol Genet. 2004;13: 1205-12.

31. Hinney A, Scherag S, Hebebrand J. Genetic findings in anorexia and bulimia nervosa. Prog Mol Biol Transl Sci. 2010;94:241-70.

32. Hebebrand J, Remschmidt H. Anorexia nervosa viewed as an extreme weight condition: genetic implications. Hum Genet. 1995;95:1-11.

33. Muller TD, Greene BH, Bellodi L, Cavallini MC, Cellini E, Di Bella D, et al. Fat mass and obesity-associated gene (FTO) in eating disorders: evidence for association of the rs9939609 obesity risk allele with bulimia nervosa and anorexia nervosa. Obes Facts. 2012:5:408-19.

34. Scott-Van Zeeland AA, Bloss CS, Tewhey R, Bansal V, Torkamani A, Libiger O, et al. Evidence for the role of EPHX2 gene variants in anorexia nervosa. Mol Psychiatry. 2013;19(6):724-32

35. Nakabayashi K, Komaki G, Tajima A, Ando T, Ishikawa M, Nomoto J, et al. Identification of novel candidate loci for anorexia nervosa at 1q41 and 11 q22 in Japanese by a genome-wide association analysis with microsatellite markers. J Hum Genet. 2009;54:531-7.

36. Wang K, Zhang H, Bloss CS, Duvvuri V, Kaye W, Schork NJ, et al. A genomewide association study on common SNPs and rare CNVs in anorexia nervosa. Mol Psychiatry. 2011;16:949-59.

37. Boraska V, Franklin CS, Floyd JA, Thornton LM, Huckins LM, Southam L, et al. A genome-wide association study of anorexia nervosa. Mol Psychiatry. 2014 19(10):1085-94.

38. Meigs JB, Shrader $P$, Sullivan LM, McAteer JB, Fox CS, Dupuis J, et al. Genotype Score in Addition to Common Risk Factors for Prediction of Type 2 Diabetes. N Eng J Med. 2008;359:2208-19.

39. Wei Z, Wang K, Qu HQ, Zhang H, Bradfield J, Kim C, et al. From disease association to risk assessment: an optimistic view from genome-wide association studies on type 1 diabetes. PLoS Genet. 2009;5:e1000678.

40. Wacholder S, Hartge P, Prentice R, Garcia-Closas M, Feigelson HS, Diver WR, et al. Performance of Common Genetic Variants in Breast-Cancer Risk Models. N Engl J Med. 2010;362:986-93.

41. Makowsky R, Pajewski NM, Klimentidis YC, Vazquez Al, Duarte CW, Allison DB, et al. Beyond Missing Heritability: Prediction of Complex Traits. PLoS Genet. 2011;7:e1002051.

42. So H-C, Kwan Johnny SH, Cherny Stacey S, Sham Pak C. Risk Prediction of Complex Diseases from Family History and Known 
Susceptibility Loci, with Applications for Cancer Screening. Am J Hum Genet. 2011;88:548-65.

43. Lubke GH, Hottenga JJ, Walters R, Laurin C, de Geus EJC, Willemsen G, et al. Estimating the Genetic Variance of Major Depressive Disorder Due to All Single Nucleotide Polymorphisms. Biol Psychiatry. 2012;72:707-9.

44. Wei Z, Wang W, Bradfield J, Li J, Cardinale C, Frackelton E, et al. Large sample size, wide variant spectrum, and advanced machine-learning technique boost risk prediction for inflammatory bowel disease. Am J Hum Genet. 2013;92:1008-12.

45. Belgard TG, Jankovic I, Lowe JK, Geschwind DH. Population structure confounds autism genetic classifier. Mol Psychiatry. 2014;19:405-7.

46. Skafidas E, Testa R, Zantomio D, Chana G, Everall IP, Pantelis C. Predicting the diagnosis of autism spectrum disorder using gene pathway analysis. Mol Psychiatry. 2014;19:504-10.

47. Manolio TA. Genomewide association studies and assessment of the risk of disease. N Engl J Med. 2010;363:166-76.

48. Green ED, Guyer MS. Charting a course for genomic medicine from base pairs to bedside. Nature. 2011;470:204-13.

49. Manolio TA, Green ED. Leading the way to genomic medicine. Am J Med Genet C Semin Med Genet. 2014;166C:1-7.

50. Kruppa J, Ziegler A, Konig IR. Risk estimation and risk prediction using machine-learning methods. Hum Genet. 2012;131:1639-54.

51. Kraft P, Wacholder S, Cornelis MC, Hu FB, Hayes RB, Thomas G, et al. Beyond odds ratios-communicating disease risk based on genetic profiles. Nat Rev Genet. 2009;10:264-9.

52. Bradley AP. The use of the area under the roc curve in the evaluation of machine learning algorithms. Pattern Recognit. 1997;30:1145-59.

53. Kaye WH, Devlin B, Barbarich N, Bulik CM, Thornton L, Bacanu SA, et al. Genetic analysis of bulimia nervosa: methods and sample description. Int J Eat Disord. 2004;35:556-70.

54. Kaye WH, Lilenfeld LR, Berrettini WH, Strober M, Devlin B, Klump KL, et al. A search for susceptibility loci for anorexia nervosa: methods and sample description. Biol Psychiatry. 2000;47:794-803.

55. Pinheiro AP, Bulik CM, Thornton LM, Sullivan PF, Root TL, Bloss CS, et al. Association study of 182 candidate genes in anorexia nervosa. Am J Med Genet B Neuropsychiatr Genet. 2010;153B:1070-80.

56. Elia J, Glessner JT, Wang K, Takahashi N, Shtir CJ, Hadley D, et al. Genomewide copy number variation study associates metabotropic glutamate receptor gene networks with attention deficit hyperactivity disorder. Nat Genet. 2012;44:78-84.

57. Glessner JT, Reilly MP, Kim CE, Takahashi N, Albano A, Hou C, et al. Strong synaptic transmission impact by copy number variations in schizophrenia. Proc Natl Acad Sci U S A. 2010;107:10584-9.

58. Glessner JT, Wang K, Cai G, Korvatska O, Kim CE, Wood S, et al. Autism genome-wide copy number variation reveals ubiquitin and neuronal genes. Nature. 2009;459:569-73.

59. Wang K, Zhang H, Ma D, Bucan M, Glessner JT, Abrahams BS, et al. Common genetic variants on 5 p14.1 associate with autism spectrum disorders. Nature. 2009;459:528-33.

60. Durstenfeld R. Algorithm-235 - Random Permutation [G6]. Commun Acm. 1964;7:420-0.

61. Wray NR, Yang J, Hayes BJ, Price AL, Goddard ME, Visscher PM. Pitfalls of predicting complex traits from SNPs. Nat Rev Genet. 2013;14:507-15.

62. Purcell $S$, Neale B, Todd-Brown K, Thomas L, Ferreira MA, Bender D, et al. PLINK: a tool set for whole-genome association and population-based linkage analyses. Am J Hum Genet. 2007;81:559-75.

63. Friedman J, Hastie T, Tibshirani R. Regularization Paths for Generalized Linear Models via Coordinate Descent. J Stat Softw. 2010;33:1-22.

64. Galatzer-Levy IR, Karstoft KI, Statnikov A, Shalev AY. Quantitative forecasting of PTSD from early trauma responses: a Machine Learning application. J Psychiatr Res. 2014:59:68-76.

65. Li C, Yang C, Gelernter J, Zhao H. Improving genetic risk prediction by leveraging pleiotropy. Hum Genet. 2014;133:639-50.

66. Fan J, LV J. Sure independence screening for ultrahigh dimensional feature space. J R Stat Soc Series B Stat Methodol. 2008:70:849-83.

67. Cook NR. Use and misuse of the receiver operating characteristic curve in risk prediction. Circulation. 2007;115:928-35.

68. Bradfield JP, Qu HQ, Wang K, Zhang H, Sleiman PM, Kim CE, et al. A genome-wide meta-analysis of six type 1 diabetes cohorts identifies multiple associated loci. PLoS Genet. 2011;7:e1002293.
69. Jostins L, Ripke S, Weersma RK, Duerr RH, McGovern DP, Hui KY, et al. Host-microbe interactions have shaped the genetic architecture of inflammatory bowel disease. Nature. 2012;491:119-24.

70. Schizophrenia Working Group of the Psychiatric Genomics Consortium. Biological insights from 108 schizophrenia-associated genetic loci. Nature. 2014:511:421-7.

71. O'Donovan MC, Craddock N, Norton N, Williams H, Peirce T, Moskvina V, et al. Identification of loci associated with schizophrenia by genome-wide association and follow-up. Nat Genet. 2008:40:1053-5.

\section{Submit your next manuscript to BioMed Central and we will help you at every step:}

- We accept pre-submission inquiries

- Our selector tool helps you to find the most relevant journal

- We provide round the clock customer support

- Convenient online submission

- Thorough peer review

- Inclusion in PubMed and all major indexing services

- Maximum visibility for your research

Submit your manuscript at www.biomedcentral.com/submit
Biomed Central 